\title{
Use of Bevacizumab in Metastatic Colorectal Cancer
}

\section{Report from the Mexican Opinion and Analysis Forum on Colorectal Cancer Treatment with Bevacizumab (September 2009)}

Juan W. Zinser-Sierra, ${ }^{1}$ Saúl Rodríguez-Ramírez, ${ }^{2}$ Ricardo Villalobos-Valencia ${ }^{3}$ and Marcelino Ramírez-Márquez, ${ }^{4}$ on behalf of the Mexican Oncologist Participants at the Cantalagua Colorectal Cancer Forum

1 Instituto Nacional de Cancerología (INCan), Mexico City, Mexico

2 Hospital de Oncologìa (CMNSXXI), Mexico City, Mexico

3 UMAE Hospital de Oncología, Mexico City, Mexico

4 Hospital Cima Chihuahua, Chihuahua, Mexico

\section{Contents}

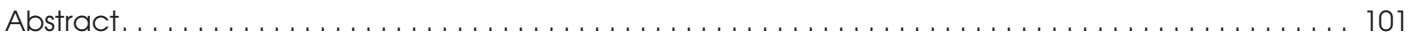

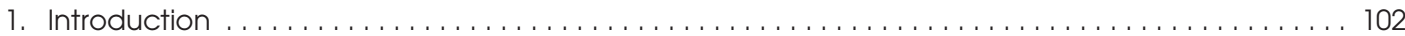

2. Predictive and Prognostic Biomarkers in Colorectal Cancer . . . . . . . . . . . . . . . . . . . . 103

3. Angiogenesis and Vascular Endothelial Growth Factor . . . . . . . . . . . . . . . . . . . . . . . . 105

4. Bevacizumab and Metastatic Colorectal Cancer . . . . . . . . . . . . . . . . . . . . . . . . . . . . 105

5. Surgical Resection of Metastatic Disease and Perioperative Chemotherapy. . . . . . . . . . . . . . . 107

6. New Treatment Paradigms in Colorectal Cancer . . . . . . . . . . . . . . . . . . . . . . . . 108

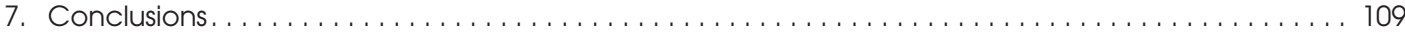

\section{Abstract}

Colorectal cancer is one of the most common cancers worldwide, and although associated mortality rates in South American countries are generally among the lowest in the world, they are on the rise.

The prognosis of patients diagnosed with metastatic colorectal cancer has improved markedly over the last 12 years, increasing from 5 months with best supportive care to almost 2 years with combination chemotherapy plus bevacizumab. New prognostic and predictive biomarkers have been identified to guide therapy. Prognostic markers indicate patient survival independent of therapy and include disease stage, mutational status, and carcinoembryonic antigen. More recently, predictive markers of treatment outcomes have been identified. The most studied are mutations of the KRAS and BRAF genes, which are associated with resistance to epidermal growth factor receptortargeted therapy.

Tumor blood vessels have a number of structural and functional abnormalities that result in increased tumor vascularity and growth driven by angiogenesis. The anti-vascular endothelial growth factor (VEGF) monoclonal antibody bevacizumab, which binds to and neutralizes VEGF-A, has 
become a central part of the treatment of metastatic colorectal cancer. The addition of bevacizumab to fluorouracil (5-FU)/leucovorin, irinotecan plus bolus 5-FU/leucovorin, or irinotecan plus infusional 5-FU/leucovorin significantly improves the overall survival of patients with previously untreated metastatic colorectal cancer. In addition, a significant increase in overall survival is seen when bevacizumab is added to oxaliplatin plus infusional 5-FU/leucovorin (FOLFOX) in patients with metastatic colorectal cancer who progressed on a non-bevacizumab-containing regimen.

Although the majority of studies were performed prior to the identification of $K R A S$ and $B R A F$ as predictive biomarkers, subsequent analysis has shown the benefits of bevacizumab occur independently of the mutational status of these genes. In patients who have progressed on a bevacizumab-containing regimen, continuation of bevacizumab is significantly associated with an improved survival based on observational cohort studies. Surgical resection is recommended in patients with metastatic colorectal cancer where complete removal of tumors can be achieved. Perioperative chemotherapy using FOLFOX for 3 months before and 3 months after surgery is associated with a 9\% improvement in 3-year survival. The use of chemotherapy in patients initially deemed unresectable has produced resection rates approaching $40 \%$, and the addition of bevacizumab to chemotherapy in this setting is feasible, safe, and effective. In a study of 219 patients, the addition of bevacizumab to FOLFOX was associated with a significant increase in major or complete pathologic response compared with FOLFOX alone.

Improvements in patient survival have changed the treatment paradigm for metastatic colorectal cancer. Newer approaches view treatment not as distinct lines of therapy but as a continuum that includes personalized treatment plans offering maintenance therapy and even drug holidays between aggressive treatment periods. This approach achieves similar efficacy outcomes with reduced toxicity, and investigation of the role of bevacizumab as maintenance therapy is ongoing.

\section{Introduction}

Worldwide, colorectal cancer is the fourth most common neoplasm in men and the third most common in women. ${ }^{[1]}$ Although mortality rates from the disease in South America remain among the lowest in the world, a recent trend towards increasing mortality due to colorectal cancer has been seen in Mexico, Brazil, Chile, and Ecuador. ${ }^{[1]}$ The majority of colorectal cancer cases arise from an adenomatous polyp, which progresses into advanced adenoma with highgrade dysplasia, and finally transforms into invasive cancer. ${ }^{[2]}$ The appearance of polyps and subsequent transformation into cancerous lesions may involve both genetic and environmental factors. Colorectal cancer that is localized within the colon or has only spread to the lymph nodes is curable by surgery with or without chemotherapy, and has a 5-year survival rate of 44-93\%.[3] However, cancer that has metastasized to distant sites is generally incurable and has a 5 -year survival rate of $<10 \%$. ${ }^{[3]}$

Twenty-five years ago, few physicians were optimistic about the chances of progress in the treatment of colorectal cancer and for improved survival for patients with this disease. However, over the last decade or so, survival rates of patients with metastatic colorectal cancer have increased from 5 months with best supportive care ${ }^{[4]}$ to almost 2 years with combination chemotherapy with fluorouracil (5-FU), leucovorin plus irinotecan plus bevacizumab (figure 1). ${ }^{[7]}$ Throughout this time, a growing body of evidence 


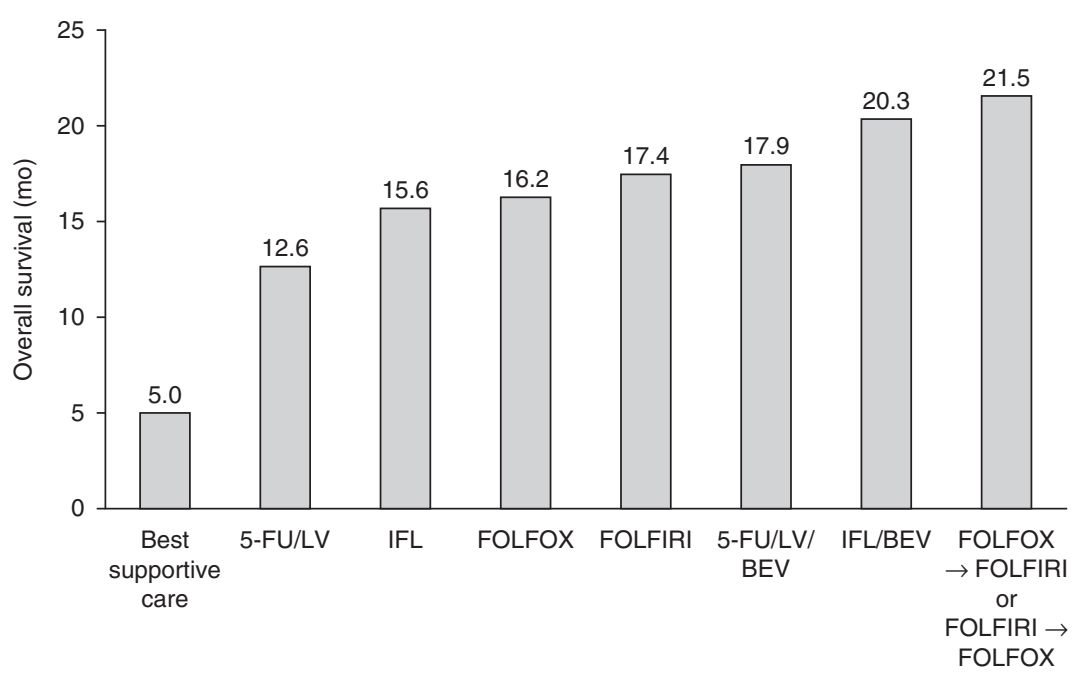

Fig. 1. Median overall survival following first-line treatment for metastatic colorectal cancer ${ }^{[4-10]} \mathbf{5}-\mathbf{F U}=$ fluorouracil; $\mathbf{B E V}=$ bevacizumab; FOLFIRI = irinotecan plus infusional fluorouracil/leucovorin; FOLFOX = oxaliplatin plus infusional fluorouracil/leucovorin; IFL=irinotecan plus bolus fluorouracil/leucovorin; LV=leucovorin.

has developed to support the importance of vascular and nutritional support for the survival of the tumor, and has ultimately led to the development of agents such as bevacizumab, which work through disruption of tumor blood flow by decreasing angiogenesis. This review is based on a series of meetings of an opinion and analysis panel in Mexico City in September 2009, and discusses a number of important issues in the treatment of colorectal cancer, including the use of prognostic and predictive biomarkers, the optimum treatment of metastatic disease, and resection of hepatic metastases.

\section{Predictive and Prognostic Biomarkers in Colorectal Cancer}

A number of prognostic and predictive indicators are used to guide therapy in patients with colorectal cancer, and the difference between the two types of markers is an important distinction to make. A prognostic marker is indicative of patient survival independent of treatment, whereas a predictive marker is indicative of therapeutic efficacy.

Colorectal cancer may arise from two distinct mutational pathways. Approximately $15 \%$ of tu- mors display microsatellite instability (MSI) mutations, giving rise to a phenotype of tumors termed high-frequency MSI (MSI-H). ${ }^{[1]}$ However, the majority $(\sim 85 \%)$ of colorectal cancers arise through chromosomal instability and have genetic alterations involving loss of heterozygosity, chromosomal amplifications, and chromosomal translocations. ${ }^{[11]}$ These tumors, termed microsatellite-stable (MSS) tumors, present and behave in a similar way to low-frequency MSI (MSI-L) tumors. Compared with MSS and MSI-L tumors, MSI-H tumors are more frequently right-sided, high-grade, and mucinous types. ${ }^{[11]}$ They are also likely to have a larger primary tumor at diagnosis, but are more frequently node negative. Patients with MSI-H tumors have a better long-term prognosis compared with similarly staged MSS tumors. MSI might be a predictive marker, as patients with MSI-H colon cancers gain no benefit from 5-FU-based adjuvant chemotherapy. ${ }^{[12]}$

Elevated carcinoembryonic antigen (CEA) prior to surgical resection is a poor prognostic indicator; a serum level of $5 \mathrm{ng} / \mathrm{mL}$ is considered a standard cut-off. ${ }^{[13]}$ While in the past CEA levels have been used in conjunction with overall stage to define a subset of patients in whom postoperative chemotherapy is required, current 
guidelines recommend that CEA only be used to indicate disease recurrence following resection. ${ }^{[14]}$ Abnormalities of the tumor suppressor gene p53, situated on chromosome $17 \mathrm{p}$, are associated with the development of colorectal cancer, but the current value of using $p 53$ as a prognostic marker in colorectal cancer is unclear. ${ }^{[11]}$ For example, an increased risk of mortality was seen with the presence of $p 53$ mutation in resected stage II and III colon cancer in a retrospective review. ${ }^{[1]}$ However, expression of $p 53$ was associated with favorable survival in stage III but not stage II disease in a study by the Southwest Oncology Group..$^{[15]}$

The RAS and RAF families of proteins act as transducers and integrators of signals from a number of surface growth factors with downstream effects on subsequent transducers, such as PI3K and MAPK. Mutations of KRAS occur in approximately $40 \%$ of colorectal cancer specimens, ${ }^{[16]}$ and although these mutations do not appear to be a prognostic factor in colorectal cancer, they do confer resistance to epidermal growth factor receptor (EGFR)-targeted therapy. For example, in the CRYSTAL trial (see table I for full trial names), in which patients with metastatic EGFR-expressing colorectal cancer received irinotecan plus infusional 5-FU/leucovorin (FOLFIRI) with or without cetuximab, the benefit of the anti-EGFR monoclonal antibody was confined to patients without KRAS mutations. ${ }^{[17]}$ Based on these and other findings, the European Medicines Agency approved the EGFR inhibitor

Table I. Trial names

\begin{tabular}{|c|c|}
\hline Acronym & Trial name \\
\hline$\overline{\mathrm{BEAT}}$ & Bevacizumab Expanded Access Trial \\
\hline $\mathrm{BICC}-\mathrm{C}$ & $\begin{array}{l}\text { Bolus, Infusional, or Capecitabine with Camptosar- } \\
\text { Celecoxib }\end{array}$ \\
\hline BOND-2 & Bowel Oncology with Cetuximab Antibody \\
\hline BRiTE & $\begin{array}{l}\text { Bevacizumab Regimens: Investigation of Treatment } \\
\text { Effects and Safety }\end{array}$ \\
\hline CAIRO & $\begin{array}{l}\text { Capecitabine, Irinotecan, and Oxaliplatin in Advanced } \\
\text { Colorectal Cancer }\end{array}$ \\
\hline CRYSTAL & $\begin{array}{l}\text { Cetuximab Combined With Irinotecan in First-Line } \\
\text { Therapy for Metastatic Colorectal Cancer }\end{array}$ \\
\hline OPTIMOX & Optimized Leucovorin-Fluorouracil-Oxaliplatin \\
\hline
\end{tabular}

panitumumab for use in patients with wild-type $K R A S$ only. ${ }^{[18]} B R A F$ mutations occur less frequently, but are also associated with reduced response to EGFR inhibitors. The BRAF-V600E mutation, in particular, impairs the therapeutic effects of cetuximab and panitumumab. ${ }^{[19]}$ Consensus guidelines recommend that the presence of $K R A S$ mutations be determined in all patients diagnosed with stage IV colorectal cancer. ${ }^{[14]}$

There are currently no suitable predictive biomarkers for bevacizumab treatment. ${ }^{[20-23]}$

It was originally thought that markers of tumor angiogenesis could be used to predict clinical outcome to bevacizumab therapy; however, vascular endothelial growth factor (VEGF) expression and microvessel density do not predict treatment outcome in patients with colorectal cancer. ${ }^{\left[{ }^{[2]}\right.}$ In addition, thrombospondin expression within the tumor does not predict response to bevacizumab. ${ }^{22]}$

Angiopoietin-2 is a key regulator of VEGF function, ${ }^{[24]}$ and Goede et al. ${ }^{[25]}$ investigated whether angiopoietin-2 was a candidate biomarker for predicting outcome in patients with metastatic colorectal cancer treated with bevacizumab. Serum angiopoietin-2 was significantly elevated in patients with metastatic colorectal cancer versus healthy subjects. Patients with stage IV colorectal cancer had significantly higher serum angiopoietin-2 levels versus patients with stage I-III colorectal cancer or healthy controls $(3.9 \mathrm{vs} 2.3 \mathrm{ng} / \mathrm{mL}$, $\mathrm{p}=0.001$; and 3.9 vs $2.4 \mathrm{ng} / \mathrm{mL}, \mathrm{p}=0.006$, respectively). Low serum angiopoietin-2 levels were associated with a significantly better response rate $(82 \%$ vs $31 \%$; p < 0.01$)$ compared with high serum angiopoietin-2 levels in patients receiving bevacizumab-based regimens. A prolonged median progression-free survival (14.1 vs 8.5 months; $\mathrm{p}<0.01)$ and a $91 \%$ reduction in death $(\mathrm{p}<0.05)$ was also observed. ${ }^{[25]}$

In a phase II study examining the efficacy of FOLFIRI plus bevacizumab in patients previously untreated for metastatic colorectal cancer, elevated baseline interleukin-8 levels were associated with a shorter median progression-free survival (11 vs 15.1 months; $\mathrm{p}=0.03){ }^{[26]}$ Basic fibroblast growth factor, placental growth factor, and hepatic growth factor were all elevated before disease progression. ${ }^{[26]}$ 
Further investigation into the predictive molecular markers for clinical outcome to bevacizumab is required.

\section{Angiogenesis and Vascular Endothelial Growth Factor}

Blood vessels supplying tumors have a number of abnormalities in their structure and function. ${ }^{[27]}$ Like normal blood vessels, those in solid tumors are composed of endothelial cells, mural cells, and a basement membrane. However, tumor vessels exhibit abnormalities in each of the structural components. ${ }^{[27]}$ In addition, they are irregularly shaped, poorly organized, and lack the arteriole-capillary-venule hierarchy. The secretion of certain factors alters the balance between endothelial cell proliferation and apoptosis, resulting in increased cell division and stimulation of the formation of new vessels through angiogenesis. ${ }^{[28,29]}$ Co-option of existing blood vessels or incorporation of bone marrow progenitor cells into existing vessels also increases tumor blood supply. ${ }^{[30]}$ Structural abnormalities of the endothelial layer of tumor blood vessels reduce normal barrier function and make them prone to leaking. ${ }^{[31]}$ This increases interstitial fluid pressure, compromising blood flow to the affected area, and has implications for drug delivery to the tumor. ${ }^{[32]}$ Impaired barrier function also increases the extravasation of tumor cells, and thus metastasis and growth factors produced or released by blood monocytes/macrophages contribute to tumor progression. ${ }^{[27,33]}$ This impaired endothelial function increases the risk of hemorrhage in the tumor and has implications for patients undergoing surgery. ${ }^{[27]}$

VEGF is highly expressed on the endothelial cells of blood vessels supplying tumors and promotes the growth of endothelial cells in arteries, veins, and lymphatic vessels. ${ }^{[34]}$ In addition, it stimulates angiogenesis in vivo, ${ }^{[35]}$ promotes vascular permeability and capillary leak, ${ }^{[36]}$ and induces the expression of molecules that control the adhesion of leukocytes in models of inflammation. ${ }^{[37]}$ These effects occur through binding of VEGF to VEGF receptor-2 (also known as Flk-1 or KDR). ${ }^{[38]}$ Bevacizumab is a humanized mono- clonal antibody that binds to and neutralizes VEGF-A. It has a half-life of approximately 17-21 days and does not elicit an antibody response in clinical trials. ${ }^{[39]}$ Bevacizumab has been investigated for the treatment of a range of tumor types and is an important factor in the treatment of metastatic colorectal cancer.

\section{Bevacizumab and Metastatic Colorectal Cancer}

Bevacizumab improves overall survival in patients with metastatic colorectal cancer when used in conjunction with 5-FU-based chemotherapy regimens. The majority of studies were conducted prior to testing for the predictive markers discussed in section 2; however, subsequent analyses show that benefit with bevacizumab occurs irrespective of $K R A S, B R A F$, or p53 status. ${ }^{[40]} \mathrm{A}$ pooled analysis of phase II studies showed a median survival of 17.9 months in patients receiving 5-FU/leucovorin plus bevacizumab compared with 14.6 months with 5-FU/leucovorin or irinotecan $(\mathrm{p}=0.008) .{ }^{[8]}$ In a pivotal study, the addition of bevacizumab to irinotecan as first-line therapy for metastatic colorectal cancer was associated with a significant increase in median overall survival, progression-free survival, and duration of response (figure 2) ${ }^{[7]}$ In the BICC-C study, patients receiving FOLFIRI, modified irinotecan, or capecitabine plus irinotecan experienced median overall survival of $23.1,17.6$, and 18.9 months, respectively. A protocol change allowed for the addition of bevacizumab mid-trial, and in patients receiving this drug, median overall survival was not reached with FOLFIRI plus bevacizumab, but was reached at 19.2 months with modified irinotecan plus bevacizumab. No direct comparisons between regimens with or without bevacizumab were made. ${ }^{[41]}$ When bevacizumab was added to oxaliplatin-containing chemotherapy regimens, a significant improvement in progressionfree survival ( 9.4 vs 8.0 months; $p=0.0023$ ), but not overall survival (21.3 vs 19.9 months; $p=0.077$ ), was seen. ${ }^{[42]}$ As second-line therapy in patients who had progressed on a non-bevacizumab-containing regimen, the addition of bevacizumab to oxaliplatin plus infusional 5-FU/leucovorin 


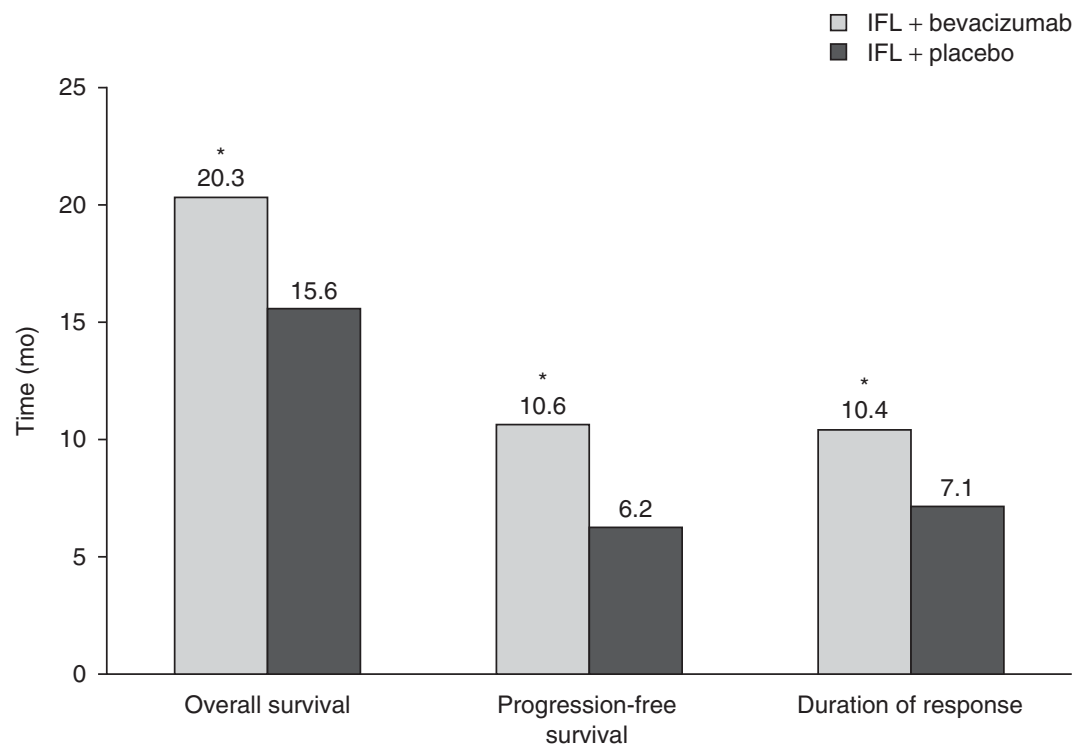

Fig. 2. Median overall survival, progression-free survival, and duration of response in 813 patients with previously untreated metastatic colorectal cancer treated with irinotecan plus bolus fluorouracil/leucovorin (IFL) and randomized to treatment with bevacizumab or placebo in a multicenter controlled study. ${ }^{[7] *} \mathrm{p}<0.001$.

(FOLFOX) 4 was associated with a significant increase in overall survival (12.9 vs 10.8 months; $\mathrm{p}=0.0011){ }^{[43]}$

With respect to adverse events associated with bevacizumab, the elderly may experience an increased risk of stroke and other arterial events, and the drug is associated with impaired wound healing and, rarely, gastrointestinal perforation. ${ }^{[14]}$

Patients with metastatic colorectal cancer that have progressed on a bevacizumab-containing regimen are a challenging proposition for the oncologist. Current guidelines suggest that these patients should be treated with an anti-EGFR monoclonal antibody; however, as discussed in section 2, these agents are only effective in patients with wild-type KRAS. ${ }^{[14]}$ The BOND-2 study was a phase II trial that investigated the administration of cetuximab and bevacizumab in patients with irinotecan-refractory colorectal cancer $(n=43)$ that were bevacizumab naïve. ${ }^{[4]}$ Overall survival was 14.5 months in the cetuximab/ bevacizumab/irinotecan group versus 11.4 months in the cetuximab/bevacizumab group. In addition, time to tumor progression and response rates were 7.3 months and $37 \%$, respectively, in the cetuximab/bevacizumab/irinotecan groups versus 4.9 months and $20 \%$ in patients who received cetuximab/bevacizumab. The toxicity profile of the cetuximab/bevacizumab/irinotecan combination is comparable with the toxicities seen with the individual agents. ${ }^{[44]}$ The CAIRO-2 study was a phase III trial of capecitabine, oxaliplatin plus bevacizumab, with or without cetuximab. ${ }^{[45]} \mathrm{A}$ significant decrease in progression-free survival was observed in the cetuximab/bevacizumab arm versus the control arm (9.8 vs 10.7 months; $\mathrm{p}=0.019$ ). Diarrhea was the most frequent grade 3-4 toxicity, and an increase in skin toxicity was observed in the cetuximab/bevacizumab group..$^{[45]}$

The observational cohort BRiTE study showed that changing the chemotherapy regimen but continuing bevacizumab therapy after disease progression was associated with a significantly greater overall survival than continuing chemotherapy without bevacizumab (hazard ratio 0.48 ; $\mathrm{p}<0.001) .{ }^{[46]}$ While encouraging, these data are from an observational study and will need to be validated in an ongoing, prospective, randomized study. 


\section{Surgical Resection of Metastatic Disease and Perioperative Chemotherapy}

Surgical resection offers the chance of long-term survival in patients who present with colorectal cancer and hepatic or pulmonary metastases, with a $25-35 \%$ rate of 5-year survival observed after complete resection. ${ }^{[47]}$ Important aspects of patient selection for surgical resection include the likelihood of achieving negative surgical margins and the maintenance of adequate liver reserve. ${ }^{[14]}$ The aim of surgical resection in this setting is curative, and the procedure should only be considered in patients in whom complete removal of known tumors is deemed possible. ${ }^{[14]}$ European guidelines recommend the use of perioperative chemotherapy in patients with resectable liver metastases, administered for 3 months before and 3 months after surgery. ${ }^{[47]}$ The use of perioperative FOLFOX4 improved 3-year progression-free survival by $7.3 \%$ in all randomized patients and by $9.2 \%$ in all patients undergoing resection in the European Organization for Research and Treatment of Cancer Intergroup trial 40983. ${ }^{[48]}$

Initially, unresectable metastases may be rendered resectable following the administration of systemic chemotherapy with or without procedures of liver hypertrophy or embolization. Use of first-line combination chemotherapy in this setting has been shown to allow resection rates of up to $40 \%$ in patients who were not previously eligible for surgery. ${ }^{[49]}$ As discussed in section 4, the addition of bevacizumab to combination chemotherapy improves outcomes in patients with metastatic disease, and the use of this strategy prior to metastasectomy has increased. The safety of preoperative chemotherapy on surgical outcomes has been a matter of some concern, particularly with regard to wound-healing complications following preoperative bevacizumab therapy. However, a number of recent studies have shown that secondary resection following bevacizumab therapy is feasible, safe, and effective. ${ }^{[50-53]}$ A nonrandomized study in which 56 patients with potentially curable metastatic colorectal cancer received preoperative treatment with capecitabine/oxaliplatin (XELOX) plus bevacizumab for six cycles with bevacizumab omitted for the final cycle showed no increases in the rate of surgical or wound-healing complications or bleeding severity with this regimen. ${ }^{[51]}$ Furthermore, neoadjuvant bevacizumab did not affect liver regeneration after resection. ${ }^{[51]}$ In the First BEAT, 225 of 1965 patients underwent curative hepatic metastasectomy, of whom complete resection with no residual disease was achieved in 173 patients. ${ }^{[52]}$ First BEAT collected safety data (primary endpoint) prospectively from patients undergoing surgery and hence, is the largest set of data using targeted therapy in this population. Patients in this study received fluoropyrimidinebased chemotherapy plus bevacizumab, with bevacizumab therapy ceasing a median of 64 days before surgery. A 2-year overall survival rate of $89 \%$ was seen in patients undergoing surgical resection with no residual disease. ${ }^{[52]}$ The incidence of grade 3 or 4 bleeding, wound-healing complications, and gastrointestinal perforation were $2.7 \%, 1.8 \%$, and $2.2 \%$, respectively, with no increase seen compared with historic controls. ${ }^{[52]}$ In the NO16966 trial (study of Xeloda ${ }^{\circledR}$ [capecitabine] as a first-line therapy in patients with metastatic colorectal cancer), 1400 patients were randomized to receive XELOX or FOLFOX4 with or without bevacizumab in a $2 \times 2$ design. ${ }^{[52]}$ NO16966 was designed to demonstrate the noninferiority of XELOX to FOLFOX4, and the data were collected retrospectively. Curative resection was performed in 44 patients randomized to bevacizumab and 34 patients receiving chemotherapy plus placebo. Two-year overall survival was $90.9 \%$ in bevacizumab recipients compared with $82.3 \%$ in those receiving placebo, but, more importantly, no increased incidence of bleeding, wound-healing complications, or gastrointestinal perforation was seen in bevacizumab recipients. ${ }^{[52]}$ In a study that included 219 patients, the addition of bevacizumab to FOLFOX as preoperative chemotherapy in patients undergoing hepatic resection produced a significant increase in response compared with FOLFOX alone. ${ }^{[53]}$ A major or complete pathologic response in patients receiving between one and eight cycles of chemotherapy occurred in $67 \%$ of patients receiving 
FOLFOX plus bevacizumab compared with $48 \%$ of FOLFOX recipients $(p=0.00017)$. A longer duration of preoperative chemotherapy was associated with a higher incidence of postoperative sinusoidal injury and liver insufficiency. ${ }^{[53]}$

\section{New Treatment Paradigms in Colorectal Cancer}

The improvements in survival seen with the increased number of chemotherapeutic agents and the expanded ways in which these agents are used have brought about a changing paradigm for the treatment of colorectal cancer (figure 3 ). In the past, metastatic colorectal cancer was treated with first-line therapy until progression, at which point treatment was switched to a regimen active in the second-line setting. This pattern was con- tinued until patients had received all five active classes of drug, after which they were enrolled in clinical trials. The new approach to treatment views the choice of first and subsequent lines of treatment as a continuum rather than isolated choices. A patient's treatment may be changed prior to disease progression. Alternately, treatment may consist of aggressive treatment periods followed by breaks or maintenance therapy following treatment response. This approach to treatment may minimize the toxicity seen with individual agents. ${ }^{[54]}$ For example, oxaliplatin is associated with both acute and delayed sensory neuropathy, and a large proportion of patients receiving cumulative doses of oxaliplatin develop grade 3 cumulative neurotoxicity that may take several months to resolve. ${ }^{[54]}$ A balance between efficacy and toxicity was illustrated in the

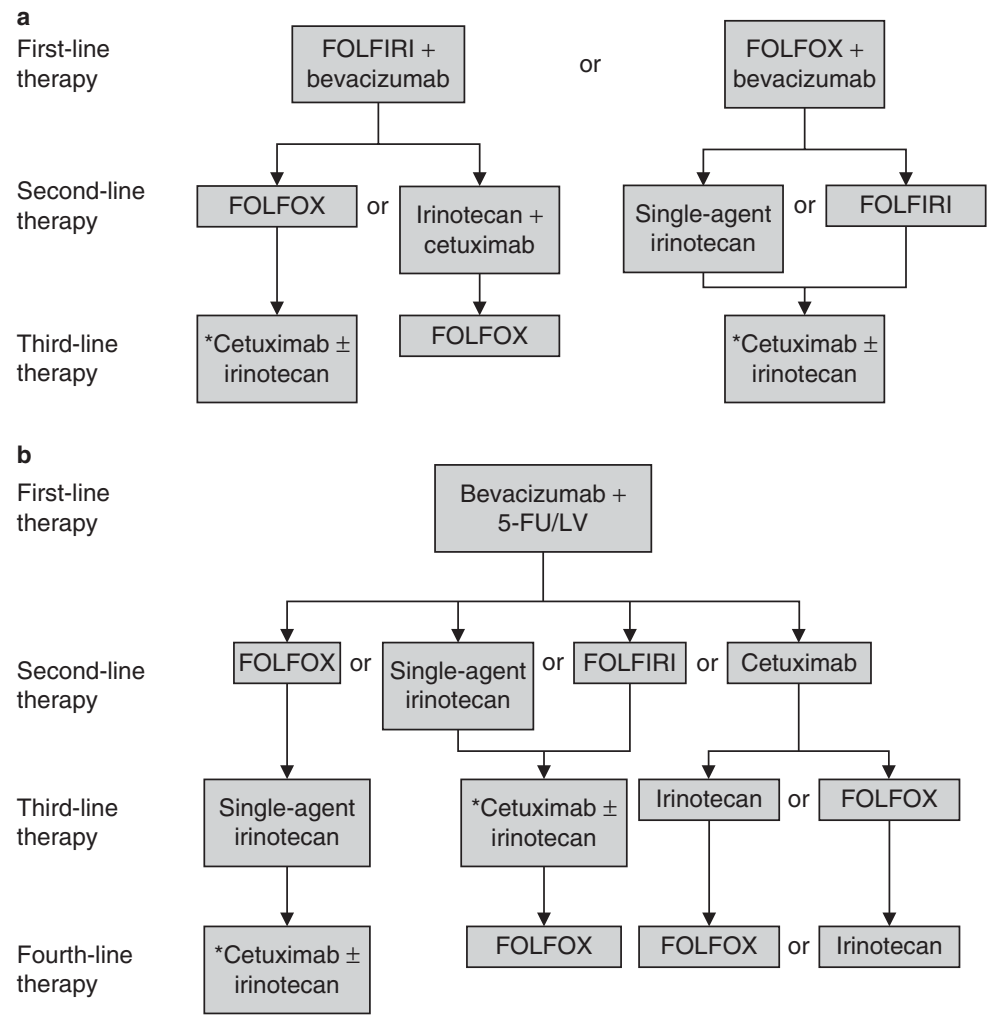

Fig. 3. Suggested treatment continuums for metastatic colorectal cancer using (a) intensive or (b) less intensive therapy. ${ }^{[54]}$ 5-FU =fluorouracil; FOLFIRI = irinotecan plus infusional fluorouracil/leucovorin; FOLFOX = oxaliplatin plus infusional fluorouracil/leucovorin; LV = leucovorin. * indicates that cetuximab may be administered alone for patients intolerant to irinotecan. Reproduced from Goldberg et al., ${ }^{[54]}$ copyright 2007, with permission of AlphaMed Press, Inc. 
OPTIMOX 1 and 2 trials. In the OPTIMOX 1 trial, patients were randomized to FOLFOX4 every 2 weeks until disease progression or highdose FOLFOX7 for six cycles followed by 5-FU/ leucovorin for 12 cycles, after which they restarted FOLFOX7. ${ }^{[5]}$ Disease duration and overall survival was similar in each group, but patients receiving FOLFOX7 had a greatly reduced incidence of peripheral neuropathy, particularly during the later stages of treatment. To refine these data, the OPTIMOX 2 study randomized patients to six cycles of modified FOLFOX7 followed by 5-FU/leucovorin maintenance until progression, or six cycles of modified FOLFOX7 followed by cessation of therapy until tumor progression; in both groups, modified FOLFOX7 was reintroduced at disease progression. ${ }^{[56]}$ The duration of disease control and progression-free survival was significantly higher in patients receiving maintenance therapy, but the overall survival was similar in both groups. Therefore, although the authors of OPTIMOX 2 concluded that chemotherapy discontinuation could be safely considered in selected patients, these results suggest maintenance therapy in metastatic colorectal cancer is important, although its role is not yet clearly defined. As a final note on the future treatment of colorectal cancer, the cytostatic mechanism of action and relatively low toxicity make bevacizumab a suitable agent for use as maintenance therapy in colorectal cancer, as it is in non-small-cell lung cancer. An ongoing, randomized, phase III study investigating this use is currently ongoing and results are eagerly awaited to further help guide the use of bevacizumab in these patients.

\section{Conclusions}

In contrast to 15 years ago, the oncologist is faced with a choice of treatments for patients with metastatic colorectal cancer and is able to predict survival of almost 2 years in these patients. Treatment should be tailored to the individual by using prognostic and predictive factors, including biomarkers. The use of these biomarkers can already assist with personalized treatment plans by, for example, identifying patients whose tumors will not respond to EGFR inhibitors, and this approach to patient care is anticipated to grow markedly in the future. Much work has been undertaken to investigate and better understand the tumor environment. The addition to chemotherapy of biologic agents such as bevacizumab that target angiogenesis, significantly improves the survival of patients with metastatic colorectal cancer independently of its biomarker status. Improved surgical techniques and use of chemotherapy plus biologic agents has also improved the odds of curative resection in patients with hepatic metastases. The prolonged survival seen with these advances in therapy has raised questions about adverse events seen with cumulative doses of chemotherapy agents and brought about new treatment paradigms, including the use of periods of intensive chemotherapy followed by drug holidays, and changing therapy early to avoid cumulative toxicity. These advances provide the platform to enable a personalized care plan formulated on an individual patient basis to further improve outcomes in metastatic colorectal cancer.

\section{Acknowledgments}

The authors would like to thank Neil Reynolds of inScience Communications, a Wolters Kluwer business, for providing medical writing support funded by Roche.

Juan W. Zinser-Sierra, MD, has received honoraria from Roche for coordinating and lecturing at meetings, and has also received honoraria from Roche for participating in symposia on colorectal cancer.

Saúl Rodríguez-Ramírez, MD, Ricardo Villalobos-Valencia, MD, and Marcelino Ramírez-Márquez, MD, have no conflicts of interest to disclose.

Participants at the Cantalagua Colorectal Cancer Forum: Ricardo Javier Almaguer-Alonso, MD; Antonio Araiza-Llaguno, MD; Leticia Bornstein, MD, MSci; Douglas Ramón CanúlRodríguez, MD; Juan Manuel Castro-Cervantes, MD; María Isabel Enríquez-Aceves, MD; Juan Pablo Flores-Gutiérrez, MD; Sergio Gainza-Lagunes, MD; Carlos García-Gutiérrez, MD; Arturo Guel-Pañola, MD; Joaquín Juárez-Durán, MD; Alejandro Juárez-Ramiro, MD; Jesús López-Hernández, MD; Germán Millán, MD; Carlos A. Ronquillo-Carreón, MD; Fernando Silva-Bravo, MD; Eduardo Urquieta-Hernández, MD; Leticia Vázquez-Cortés, MD.

\section{References}

1. Center MM, Jemal A, Smith RA, et al. Worldwide variations in colorectal cancer. CA Cancer J Clin 2009 Nov-Dec; 59 (6): 366-78 
2. Arnold CN, Goel A, Blum HE, et al. Molecular pathogenesis of colorectal cancer: implications for molecular diagnosis. Cancer 2005 Nov 15; 104 (10): 2035-47

3. O'Connell JB, Maggard MA, Ko CY. Colon cancer survival rates with the new American Joint Committee on Cancer sixth edition staging. J Natl Cancer Inst 2004 Oct 6; 96 (19): $1420-5$

4. Scheithauer W, Rosen H, Kornek GV, et al. Randomised comparison of combination chemotherapy plus supportive care with supportive care alone in patients with metastatic colorectal cancer. BMJ 1993 Mar 20; 306 (6880): 752-5

5. de Gramont A, Figer A, Seymour M, et al. Leucovorin and fluorouracil with or without oxaliplatin as first-line treatment in advanced colorectal cancer. J Clin Oncol 2000 Aug; 18 (16): 2938-47

6. Douillard JY, Cunningham D, Roth AD, et al. Irinotecan combined with fluorouracil compared with fluorouracil alone as first-line treatment for metastatic colorectal cancer: a multicentre randomised trial. Lancet 2000 Mar 25; 355 (9209): 1041-7

7. Hurwitz H, Fehrenbacher L, Novotny W, et al. Bevacizumab plus irinotecan, fluorouracil, and leucovorin for metastatic colorectal cancer. N Engl J Med 2004 Jun 3; 350 (23): 2335-42

8. Kabbinavar FF, Hambleton J, Mass RD, et al. Combined analysis of efficacy: the addition of bevacizumab to fluorouracil/leucovorin improves survival for patients with metastatic colorectal cancer. J Clin Oncol 2005 Jun 1; 23 (16): 3706-12

9. Saltz LB, Cox JV, Blanke C, et al. Irinotecan plus fluorouracil and leucovorin for metastatic colorectal cancer. Irinotecan Study Group. N Engl J Med 2000 Sep 28; 343 (13): 905-14

10. Tournigand C, Andre T, Achille E, et al. FOLFIRI followed by FOLFOX6 or the reverse sequence in advanced colorectal cancer: a randomized GERCOR study. J Clin Oncol 2004 Jan 15; 22 (2): 229-37

11. Libbutti SK, Saltz LB, Tepper JE. Colon cancer. In: DeVita VT, Lawrence TS, Rosenberg SA, editors. Cancer: principles \& practice of oncology. 8th Ed. Philadelphia (PA): Lippincott Williams and Wilkins, 2008: 1232-85

12. Ribic CM, Sargent DJ, Moore MJ, et al. Tumor microsatellite-instability status as a predictor of benefit from fluorouracil-based adjuvant chemotherapy for colon cancer. N Engl J Med 2003 Jul 17; 349 (3): 247-57

13. Compton CC, Fielding LP, Burgart LJ, et al. Prognostic factors in colorectal cancer. College of American Pathologists Consensus Statement 1999. Arch Pathol Lab Med 2000 Jul; 124 (7): 979-94

14. National Comprehensive Cancer Network. NCCN clinical practice guidelines in oncology: colon cancer [online] Available from URL: http://www.ncen.org/professionals physician_gls/PDF/colon.pdf [Accessed 2010 Apr 1]

15. Ahnen DJ, Feigl P, Quan G, et al. Ki-ras mutation and p53 overexpression predict the clinical behavior of colorectal cancer: a Southwest Oncology Group study. Cancer Res 1998 Mar 15; 58 (6): 1149-58

16. Andreyev HJ, Norman AR, Cunningham D, et al. Kirsten ras mutations in patients with colorectal cancer: the multicenter "RASCAL" study. J Natl Cancer Inst 1998 May 6; 90 (9): 675-84
17. Van Cutsem E, Kohne CH, Hitre E, et al. Cetuximab and chemotherapy as initial treatment for metastatic colorectal cancer. N Engl J Med 2009 Apr 2; 360 (14): 1408-17

18. European Medicines Agency. Vectibix: summary of product characteristics 2009 [online]. Available from URL: http:/ www.ema.europa.eu/humandocs/PDFs/EPAR/vectibix/H741-PI-en.pdf [Accessed 2010 Apr 12]

19. Di Nicolantonio F, Martini M, Molinari F, et al. Wild-type $\mathrm{BRAF}$ is required for response to panitumumab or cetuximab in metastatic colorectal cancer. J Clin Oncol 2008 Dec 10; 26 (35): 5705-12

20. Chua W, Kho PS, Moore MM, et al. Clinical, laboratory and molecular factors predicting chemotherapy efficacy and toxicity in colorectal cancer. Crit Rev Oncol Hematol. Epub 2010 Aug 16

21. De Roock W, Biesmans B, De Schutter J, et al. Clinical biomarkers in oncology: focus on colorectal cancer. Mol Diagn Ther 2009; 13 (2): 103-14

22. Jubb AM, Hurwitz HI, Bai W, et al. Impact of vascular endothelial growth factor-A expression, thrombospondin-2 expression, and microvessel density on the treatment effect of bevacizumab in metastatic colorectal cancer. J Clin Oncol 2006 Jan 10; 24 (2): 217-27

23. Murukesh N, Dive C, Jayson GC. Biomarkers of angiogenesis and their role in the development of VEGF inhibitors. Br J Cancer 2010 Jan 5; 102 (1): 8-18

24. Augustin HG, Koh GY, Thurston G, et al. Control of vascular morphogenesis and homeostasis through the angiopoietin-Tie system. Nat Rev Mol Cell Biol 2009 Mar; 10 (3): $165-77$

25. Goede V, Coutelle O, Neuneier J, et al. Identification of serum angiopoietin-2 as a biomarker for clinical outcome of colorectal cancer patients treated with bevacizumabcontaining therapy. Br J Cancer 2010 Oct 26; 103 (9): $1407-14$

26. Kopetz S, Hoff PM, Morris JS, et al. Phase II trial of infusional fluorouracil, irinotecan, and bevacizumab for metastatic colorectal cancer: efficacy and circulating angiogenic biomarkers associated with therapeutic resistance. J Clin Oncol 2010 Jan 20; 28 (3): 453-9

27. Baluk P, Hashizume H, McDonald DM. Cellular abnormalities of blood vessels as targets in cancer. Curr Opin Genet Dev 2005 Feb; 15 (1): 102-11

28. Carmeliet P, Jain RK. Angiogenesis in cancer and other diseases. Nature 2000 Sep 14; 407 (6801): 249-57

29. Verheul HM, Voest EE, Schlingemann RO. Are tumours angiogenesis-dependent? J Pathol 2004 Jan; 202 (1): 5-13

30. Holash J, Maisonpierre PC, Compton D, et al. Vessel cooption, regression, and growth in tumors mediated by angiopoietins and VEGF. Science 1999 Jun 18; 284 (5422): 1994-8

31. Hashizume H, Baluk P, Morikawa S, et al. Openings between defective endothelial cells explain tumor vessel leakiness. Am J Pathol 2000 Apr; 156 (4): 1363-80

32. Padera TP, Stoll BR, Tooredman JB, et al. Pathology: cancer cells compress intratumour vessels [abstract]. Nature 2004 Feb 19; 427 (6976): 695

33. Coussens LM, Werb Z. Inflammation and cancer. Nature 2002 Dec 19-26; 420 (6917): 860-7

34. Ferrara N, Davis-Smyth T. The biology of vascular endothelial growth factor. Endocr Rev 1997 Feb; 18 (1): 4-25 
35. Plouet J, Schilling J, Gospodarowicz D. Isolation and characterization of a newly identified endothelial cell mitogen produced by AtT-20 cells. EMBO J 1989 Dec 1; 8 (12): $3801-6$

36. Dvorak HF. Vascular permeability factor/vascular endothelial growth factor: a critical cytokine in tumor angiogenesis and a potential target for diagnosis and therapy. J Clin Oncol 2002 Nov 1; 20 (21): 4368-80

37. Melder RJ, Koenig GC, Witwer BP, et al. During angiogenesis, vascular endothelial growth factor and basic fibroblast growth factor regulate natural killer cell adhesion to tumor endothelium. Nat Med 1996 Sep; 2 (9): 992-7

38. Ferrara N. Vascular endothelial growth factor: basic science and clinical progress. Endocr Rev 2004 Aug; 25 (4): 581-611

39. Ferrara N, Hillan KJ, Gerber HP, et al. Discovery and development of bevacizumab, an anti-VEGF antibody for treating cancer. Nat Rev Drug Discov 2004 May; 3 (5): 391-400

40. Ince WL, Jubb AM, Holden SN, et al. Association of k-ras, b-raf, and p53 status with the treatment effect of bevacizumab. J Natl Cancer Inst 2005 Jul 6; 97 (13): 981-9

41. Fuchs CS, Marshall J, Mitchell E, et al. Randomized, controlled trial of irinotecan plus infusional, bolus, or oral fluoropyrimidines in first-line treatment of metastatic colorectal cancer: results from the BICC-C Study. J Clin Oncol 2007 Oct 20; 25 (30): 4779-86

42. Saltz LB, Clarke S, Diaz-Rubio E, et al. Bevacizumab in combination with oxaliplatin-based chemotherapy as firstline therapy in metastatic colorectal cancer: a randomized phase III study. J Clin Oncol 2008 Apr 20; 26 (12): 2013-9

43. Giantonio BJ, Catalano PJ, Meropol NJ, et al. Bevacizumab in combination with oxaliplatin, fluorouracil, and leucovorin (FOLFOX4) for previously treated metastatic colorectal cancer: results from the Eastern Cooperative Oncology Group Study E3200. J Clin Oncol 2007 Apr 20; 25 (12): 1539-44

44. Saltz LB, Lenz HJ, Kindler HL, et al. Randomized phase II trial of cetuximab, bevacizumab, and irinotecan compared with cetuximab and bevacizumab alone in irinotecanrefractory colorectal cancer: the BOND-2 study. J Clin Oncol 2007 Oct 10; 25 (29): 4557-61

45. Punt CJ, Tol J, Rodenburg CJ, et al. Randomized phase III study of capecitabine, oxaliplatin, and bevacizumab with or without cetuximab in advanced colorectal cancer (ACC), the CAIRO2 study of the Dutch Colorectal Cancer Group (DCCG). ASCO Annual Meeting Proceedings; 2008 May 30-Jun 3; Chicago (IL)

46. Grothey A, Sugrue MM, Purdie DM, et al. Bevacizumab beyond first progression is associated with prolonged overall survival in metastatic colorectal cancer: results from a large observational cohort study (BRiTE). J Clin Oncol 2008 Nov 20; 26 (33): 5326-34
47. Van Cutsem E, Oliveira J. Advanced colorectal cancer: ESMO clinical recommendations for diagnosis, treatment and follow-up. Ann Oncol 2009 May; 20 Suppl. 4: 61-3

48. Nordlinger B, Sorbye H, Glimelius B, et al. Perioperative chemotherapy with FOLFOX4 and surgery versus surgery alone for resectable liver metastases from colorectal cancer (EORTC Intergroup trial 40983): a randomised controlled trial. Lancet 2008 Mar 22; 371 (9617): 1007-16

49. Nordlinger B, Van Cutsem E, Gruenberger T, et al. Combination of surgery and chemotherapy and the role of targeted agents in the treatment of patients with colorectal liver metastases: recommendations from an expert panel. Ann Oncol 2009 Jun; 20 (6): 985-92

50. Cunningham D, Kretzschmar A, Berry S, et al. Efficacy and safety of surgery with curative intent in patients treated with first-line bevacizumab for mCRC: first BEAT [abstract no. 445]. American Society of Clinical Oncology 2008 Gastrointestinal Cancers Symposium; 2008 Jan 25-27; Orlando (FL)

51. Gruenberger B, Tamandl D, Schueller J, et al. Bevacizumab, capecitabine, and oxaliplatin as neoadjuvant therapy for patients with potentially curable metastatic colorectal cancer. J Clin Oncol 2008 Apr 10; 26 (11): 1830-5

52. Okines A, Puerto OD, Cunningham D, et al. Surgery with curative-intent in patients treated with first-line chemotherapy plus bevacizumab for metastatic colorectal cancer first BEAT and the randomised phase-III NO16966 trial. Br J Cancer 2009 Oct 6; 101 (7): 1033-8

53. Zorzi D, Kishi Y, Maru DM, et al. Effect of extended preoperative chemotherapy on pathologic response and postoperative liver insufficiency after hepatic resection for colorectal liver metastases [abstract no. 295]. American Society of Clinical Oncology 2009 Gastrointestinal Cancers Symposium; 2009 Jan 15-17; San Francisco (CA)

54. Goldberg RM, Rothenberg ML, Van Cutsem E, et al. The continuum of care: a paradigm for the management of metastatic colorectal cancer. Oncologist 2007 Jan; 12 (1): 38-50

55. Tournigand C, Cervantes A, Figer A, et al. OPTIMOX1: a randomized study of FOLFOX4 or FOLFOX7 with oxaliplatin in a stop-and-go fashion in advanced colorectal cancer - a GERCOR study. J Clin Oncol 2006 Jan 20; 24 (3): 394-400

56. Chibaudel B, Maindrault-Goebel F, Lledo G, et al. Can chemotherapy be discontinued in unresectable metastatic colorectal cancer? The GERCOR OPTIMOX2 study. J Clin Oncol 2009 Dec 1; 27 (34): 5727-33

Correspondence: Dr Juan W. Zinser-Sierra, Instituto Nacional de Cancerología (INCan), San Fernando No. 22, Col. Sección XVI, Delegación Tlalpan, C.P. 14080, México, D.F.

E-mail: juanwzinser@infosel.net.mx 